Supporting Information

\title{
4-Aminothiophenol-Modulated Ag Growth on Au Nanoparticles for Detection of Nitrite
}

Jiaqi Chen, ${ }^{\dagger}$ Ting Zhong, ${ }^{\dagger} \mathrm{Xi} \mathrm{Lu},{ }^{\dagger}$ Feng Zhao, ${ }^{\ddagger}$ Pan Wang, ${ }^{\dagger}$ Xue Yu, ${ }^{\dagger}$ Yuxin Yang, ${ }^{\dagger}$ Fengyun Sun, ${ }^{\dagger}$ Xiaochun $\mathrm{Wu}^{*} \S$ and Wei Feng ${ }^{*}$

† Department of Materials Engineering, School of Mechanical Engineering, Chengdu University, Chengdu 610106, Sichuan, PR China.

‡ Institute for Advanced Materials Deformation and Damage from Multi-Scale, Chengdu University, Chengdu, 610106, China.

$\S$ CAS Key Laboratory of Standardization and Measurement for Nanotechnology, CAS Center for Excellence in Nanoscience, National Center for Nanoscience and Technology, Beijing 100190, PR China.

* To whom correspondence should be addressed:

X.Wu: E-mail_wuxc@nanoctr.cn.

W.F: E-mail fengwei233@126.com 

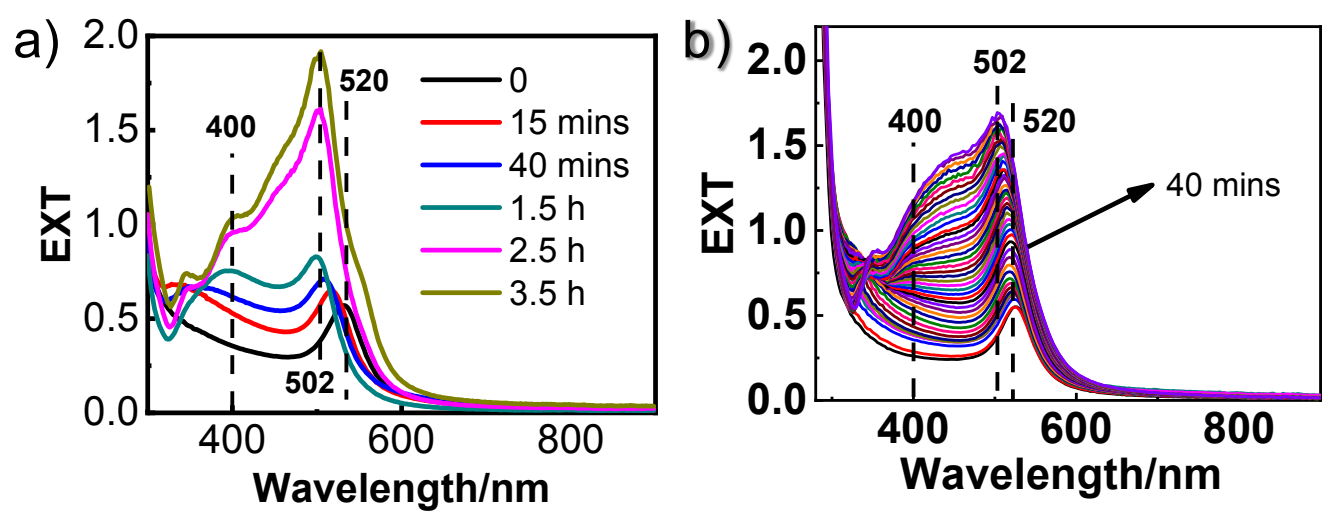

Fig. S1 The evolution of the extinction spectra of nanoparticles when the Ag shell was coated on the Au20 in the absence (a) and presence (b) of 4-ATP.

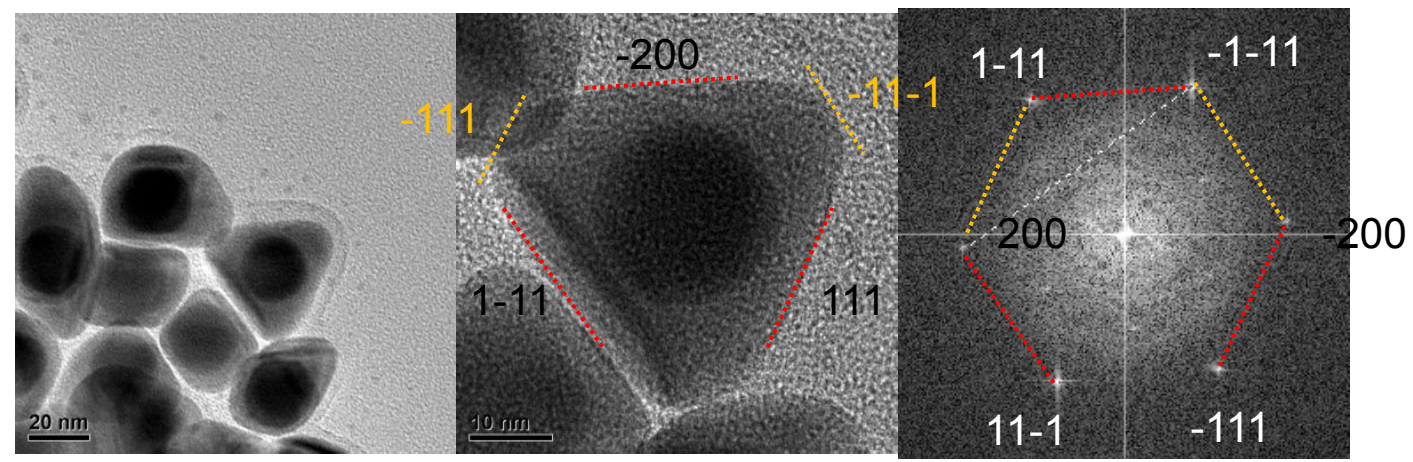

Fig. S2 The morphology analysis of Au@Ag NPs assisted with 4-ATP. TEM, the HR-TEM image and the Fast Fourier Transform (FFT) pattern of the Au@Ag NPs assisted with 10 M 4-ATP.

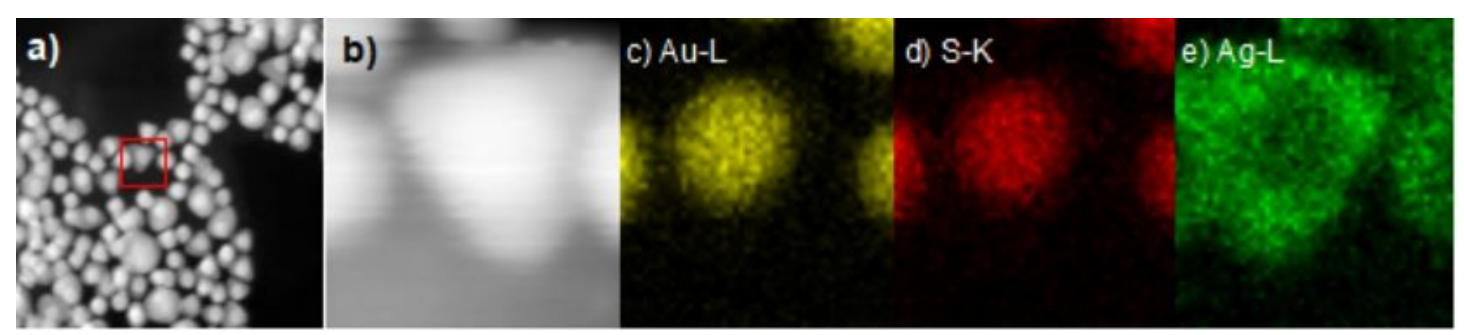

Fig. S3 The elemental mapping of the single Au@Ag NPs assisted by 4-ATP. High-angle annular dark field (HAADF)-STEM images of NPs (a) and the single NP (b). The distribution of Au-L (c), S-K (d) and Ag-L (e). 


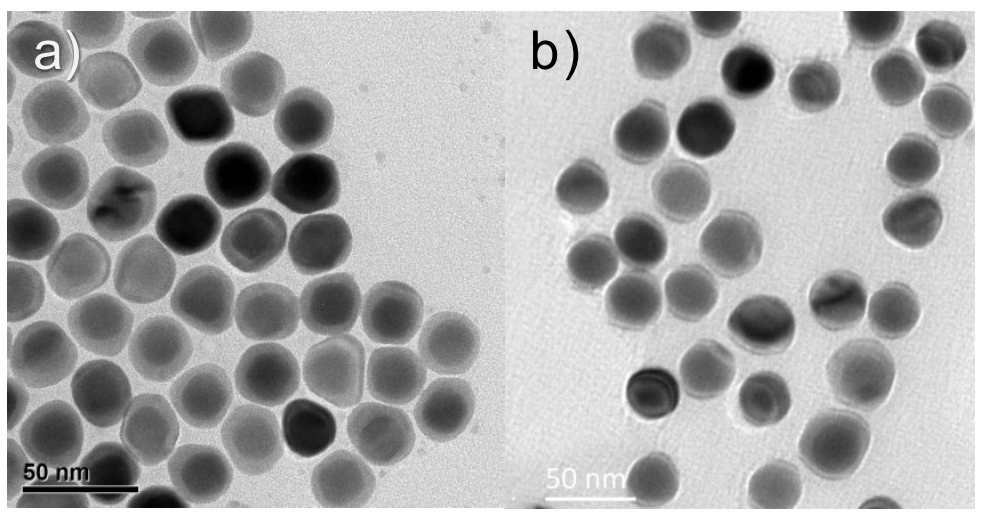

Fig. S4. The mophorlogy of Au20@Ag nanoparticles grown with $10 \mu \mathrm{M}$ 4-ATP in the absence (a) and presence (b) of $10 \mu \mathrm{M}$ nitrite in the acidic ethanol solution.
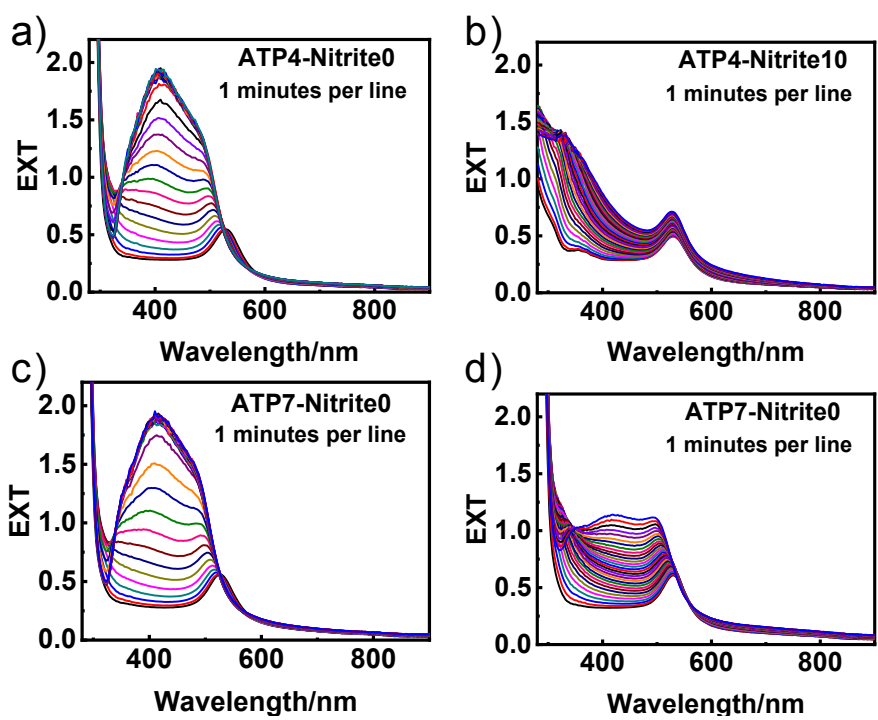

Fig. S5 The effect of [4-ATP] and nitrite on the Ag overgrowth process. The evolution of the extinction spectra of Au20@Ag nanoparticles grown from Au20 modified with 4 and $7 \mu \mathrm{M}$ 4-ATP in absence $(\mathrm{a}, \mathrm{c})$ and presence $(\mathrm{b}, \mathrm{d})$ of nitrite in the acidic ethanol solution. 
a)

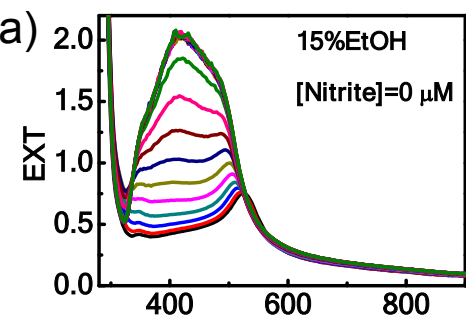

Wavelength/nm

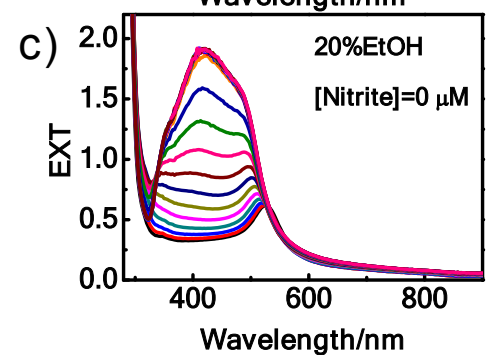

e)

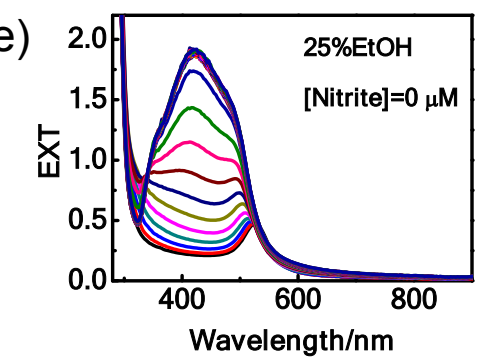

b)

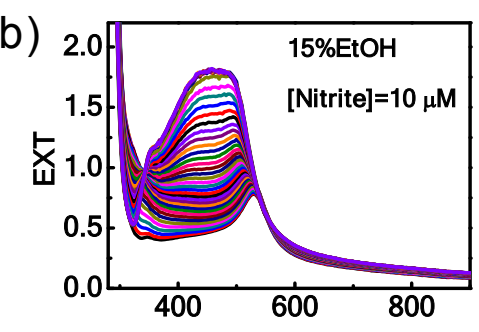

Wavelength/nm
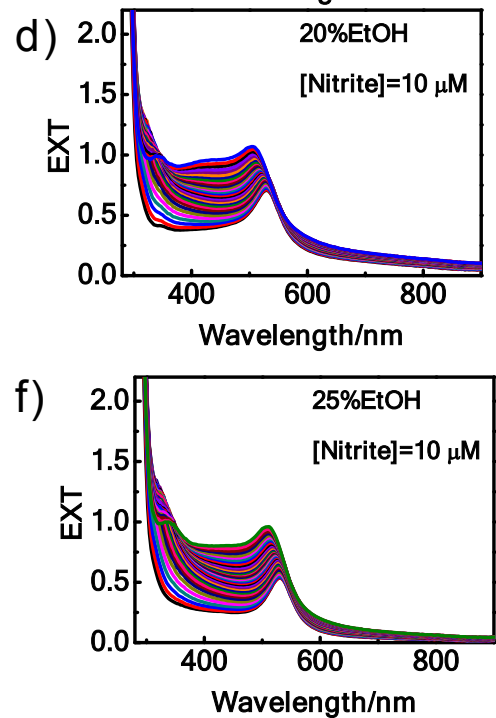

Fig.S6 The influence of the volume fraction of ethanol on the response of nitrite to the growth process. The evolution of extinction spectra of nanoparticles growing with $15 \%, 20 \%$ and $25 \%$ ethanol in the absence (a, c, e) and presence (b, d, f) of nitrite.

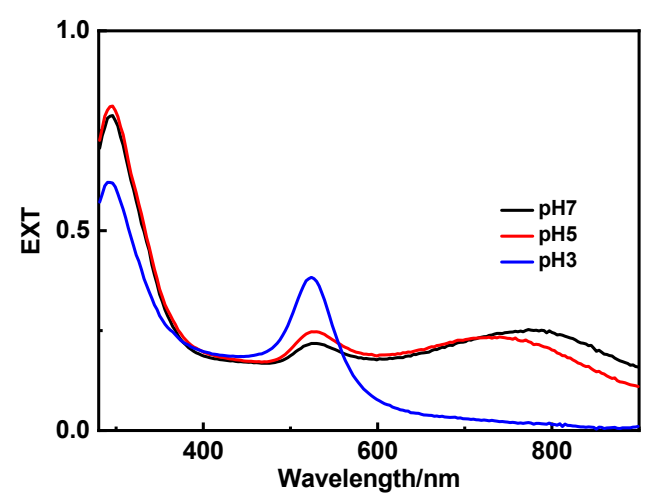

Fig. S7 The extinction spectra of Au20 after incubation at different $\mathrm{pH}$. 

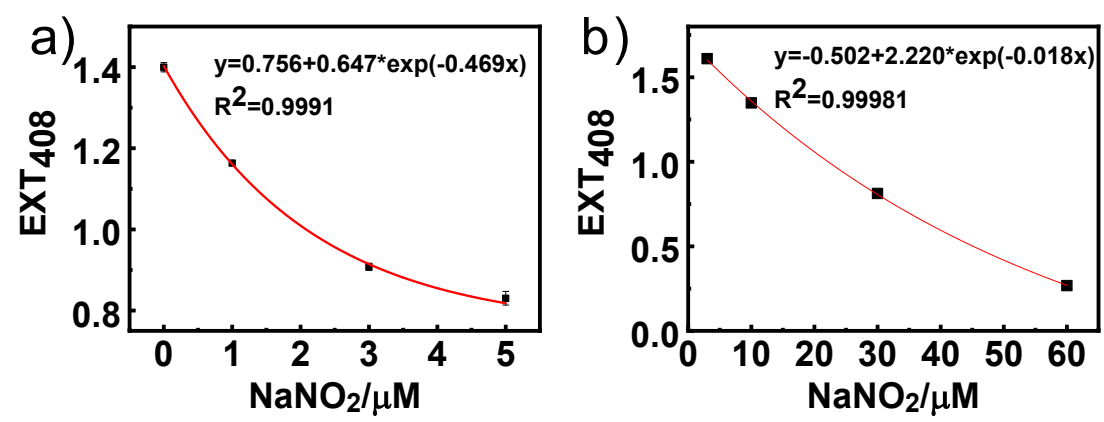

Fig. S8 EXT $_{408}$ of NPs growing for 20 minutes on Au20 after modification with (a) $10 \mu \mathrm{M}$ 4-ATP and reaction with 0-5 $\mu \mathrm{M}$ nitrite, (b) $90 \mu \mathrm{M}$ 4-ATP and reaction with 3-60 $\mu \mathrm{M}$ nitrite.

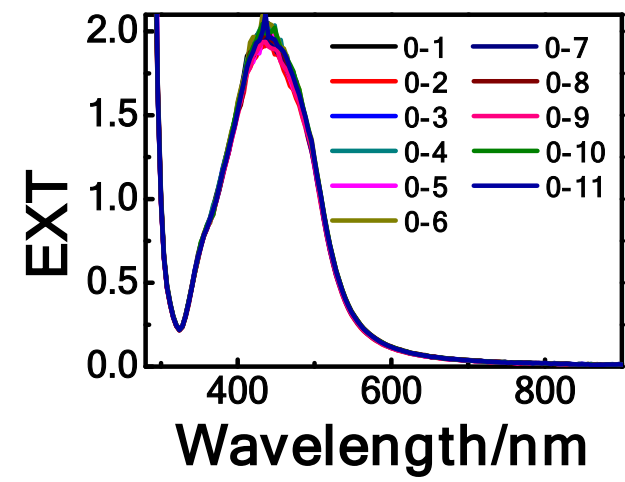

Fig. S9 The extinction spectra of 11 blank samples ([nitrite $]=0 \quad \mu$ M) after Ag coating on Au20 modified with $10 \mu \mathrm{M}$ 4-ATP for 20 minutes.

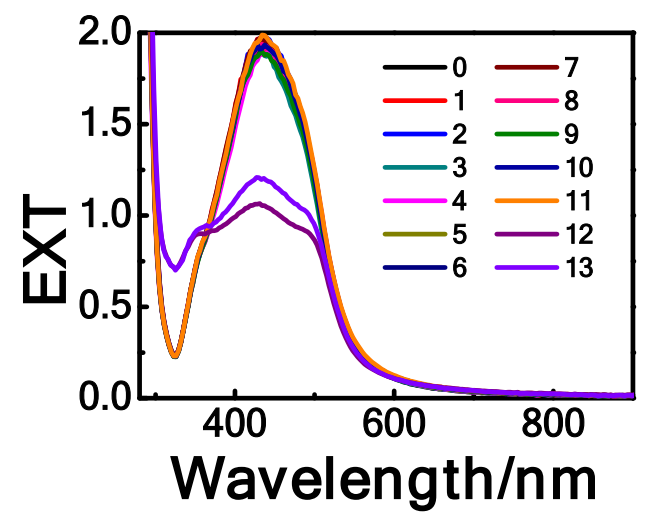


Fig. S10 Extinction spectra of nanoparticles growing for 20 minutes on Au20 after modification with $10 \mu \mathrm{M}$ 4-ATP and reaction with 11 other inorganic anions. The concentrations of 1-10 anions were $50 \mu$ M. 0:control, 1: $\mathrm{HCO}_{3}^{-}, 2: \mathrm{ClO}_{4}{ }^{-}, 3: \mathrm{Ac}^{-}, 4: \mathrm{H}_{2} \mathrm{PO}_{4}^{-}, 5: \mathrm{C}_{2} \mathrm{O}_{4}{ }^{2-}, 6: \mathrm{SO}_{4}{ }^{2-}, 7: \mathrm{CO}_{3}{ }^{2-}$, 8: $\mathrm{NH}_{4}{ }^{+}, 9: \mathrm{Br}, 10: \mathrm{F}^{-}, 11: \mathrm{NO}_{3}^{-}(10 \mu \mathrm{M}), 12: \mathrm{NO}_{2}^{-}(5 \mu \mathrm{M}), 13: \mathrm{NO}_{3}^{-}(50 \mu \mathrm{M})$

\begin{tabular}{|c|c|c|c|c|c|}
\hline Sensors & $\begin{array}{c}\text { Detection } \\
\text { mechanism }\end{array}$ & Detection range $/ \mu \mathrm{M}$ & $\mathrm{LOD} / \mu \mathrm{M}$ & Selectivity & Literature \\
\hline Au nanospheres & Aggregation & $20 \sim 35$ & Below 22 & Good & 1 \\
\hline Ag@Au nanoparticles & Redox etching & 1.0 20.0 & 0.1 & Good & 2 \\
\hline Au nanospheres & Etching & $1-1000$ & 4.5 & Good & 3 \\
\hline Au nanorods & Aggregation & $5.2-100$ & Below 5.2 & Good & 4 \\
\hline Au@Ag nanoparticles & Growth & 0 60 (Adjustable) & 0.036 & Good & This work \\
\hline
\end{tabular}

Table S1. The comparison of some reported spectrophotometric detection methods of nitrite based on plasmonic nanostructures.

\begin{tabular}{cccc|cccc}
\hline $\begin{array}{c}\text { Real } \\
\text { Sample1 }\end{array}$ & $\begin{array}{c}\text { Added } \\
/ \mu \mathrm{M}\end{array}$ & $\begin{array}{c}\text { Found } \\
/ \mu \mathrm{M}\end{array}$ & $\begin{array}{c}\text { Recovery } \\
/ \%\end{array}$ & $\begin{array}{c}\text { Real } \\
\text { Sample2 }\end{array}$ & $\begin{array}{c}\text { Added } \\
/ \mu \mathrm{M}\end{array}$ & $\begin{array}{c}\text { Found } \\
/ \mu \mathrm{M}\end{array}$ & $\begin{array}{c}\text { Recovery } \\
/ \%\end{array}$ \\
\hline Brand 1 & 3 & 1.33 & 133.00 & & 1 & 0.19 & 19.34 \\
& 5 & 4.24 & 84.80 & & 3 & 1.61 & 53.60 \\
\hline
\end{tabular}

Table S2 The determination of the added nitrite in the drinking water of Brand 1 and Brand 2.

\section{References:}

[1] Weston L. Daniel, M. S. H., Jae-Seung Lee, Chad A. Mirkin, Colorimetric nitrite and nitrate detection with gold nanoparticle probes and kinetic end points. J. Am. Chem. Soc. 2009, 131 (18), $6362-6363$.

[2] Li, T. H.; Li, Y. L.; Zhang, Y. J.; Dong, C.; Shen, Z. Y.; Wu, A. G., A colorimetric nitrite detection system with excellent selectivity and high sensitivity based on ag@au nanoparticles. Analyst 2015, 140 (4), 1076-1081.

[3] Qindeel, M.; Barani, M.; Rahdar, A.; Arshad, R.; Cucchiarini, M., Nanomaterials for the diagnosis and treatment of urinary tract infections. Nanomaterials (Basel) 2021, 11 (2).

[4] Nan Xiao, C. Y., Rapid-response and highly sensitive noncross-linking colorimetric nitrite sensor using 4-aminothiophenol modified gold nanorods. Analytical chemistry 2010, 82, 9659-3663. 